\title{
Estimativas da Composição Física das Carcaças e do Corpo Vazio de Amostras da 15a Progênie dos Rebanhos Zebu e Caracu de Sertãozinho(SP) ${ }^{1}$
}

\author{
Alexander George Razook ${ }^{2,5}$, Romeu Fernandes Nardon ${ }^{3}$, Luís Orlindo Tedeschi,5, \\ Leopoldo Andrade de Figueiredo2, Joslaine Noely dos Santos Gonçalves Cyrillo2,5, \\ Ana Claudia Ruggieri ${ }^{2,5}$
}

\begin{abstract}
RESUMO - Foram estimadas as composições corporais físicas em termos de porcentagens de músculo, gordura e osso na meia carcaça esquerda e no peso vazio estimado (PVz), de 44 machos inteiros, nascidos em 1995 e terminados em confinamento, dos rebanhos selecionados para peso aos 378 dias (P378), da Estação Experimental de Zootecnia de Sertãozinho (SP). A amostra, representando a média de cada rebanho em P378, foi de 9 animais Gir (Gi); Nelore (NeS); Nelore Controle (NeC); Caracu (Ca) e 8 Guzerá (GuS). O abate ocorreu aos 580 dias de idade e condição corporal 8 (escala 1-9). As estimativas foram obtidas por meio de equações estimadoras de quantidades (estimativa 1) de músculo, gordura e osso e porcentagens diretamente (estimativa 2) utilizando informações do corte entre a 9a e $11^{\underline{a}}$ costelas e outras características de abate. Os valores médios das duas estimativas para percentagens de músculo, gordura e osso na meia carcaça foram: Gi $(65,9,18,2$ e 15,8); GuS (64,1, 21,3 e 14,6); $\mathrm{NeC}(63,0,21,2$ e 15,7); $\mathrm{NeS}(62,6,21,3$ e 16,0) e Ca $(64,2,17,1$ e 18,6). Valores respectivos para \% em PVz foram: Gi $(41,5,11,5$ e 10,0); GuS (39,1, 13,0 e 8,9); NeC (40,0, 13,5 e 10,0); NeS $(39,4,13,4$ e 10,1) e Ca $(39,4,10,5$ e 11,4). A seleção para peso pós-desmame, visualizada na comparação entre os grupos Nelore, não promoveu alterações indiretas na composição corporal desses animais. As proporções de músculo nos outros grupos genéticos foram semelhantes aos Nelore. As porcentagens de gordura renal e pélvica, em relação ao PVz, também não foram diferentes entre animais dos vários rebanhos, contrariamente à proporção de gordura na carcaça e em PVz, que foi menor em animais Gi e Ca. As estimativas de composição corporal, resultantes dos dois tipos de equações, foram razoavelmente concordantes, diferindo somente para porcentagem de ossos em animais Ca.
\end{abstract}

Palavras-chave: seleção, composição corporal, Gir, Nelore, Guzerá, Caracu

\section{Physical Composition Estimates of Carcass and Empty Body Weight on Samples of the $15^{\text {th }}$ Progeny of Sertãozinho (SP-Brazil) Zebu and Caracu Herds}

\begin{abstract}
The body composition, in terms of percentages of muscle, fat and bone of carcass or empty body weight (EBW), was estimated in a sample comprised of 44 bulls, born in 1995, from the selected herds for weight at 378 days (W378) at Sertãozinho Experiment Station (SP-Brazil) and finished in a feedlot until slaughter. The sample of each herd, representing it's mean for W378, was 9 for each group, Gir (Gi); Nellore (NeS); Control Nellore (NeC), Caracu (Ca) and 8 for Guzerá (GuS). The slaughter was at 580 days of age with a body condition score averaging 8 (1-9 scale). The estimates were obtained either by predicting equations of muscle, fat and bone amounts (estimates 1) or percentages (estimates 2) utilizing the information of the $9^{\text {th }}-11^{\text {th }}$ rib section and other slaughter traits. Average values for the two estimates for muscle, fat and bone carcass percentages were: Gi (65.9; 18.2 and 15.8); GuS (64.1; 21.3 and 14.6); $\mathrm{NeC}(63.0 ; 21.2$ and 15.7); $\mathrm{NeS}(62.6 ; 21.3$ and 16.0) and $\mathrm{Ca}(64.2 ; 17.1$ and 18.6). Correspondent values for \% of EBW were: Gi (41.5; 11.5 and 10.0); GuS (39.1; 13.0 and 8.9); $\mathrm{NeC}(40.0 ; 13.5$ and 10.0); $\mathrm{NeS}(39.4 ; 13.4$ and 10.1) and $\mathrm{Ca}(39.4 ; 10.5$ and 11.4). The selection for post-weaning weights, observed by comparing the two Nellore groups, did not promote any change on body composition of these animals. The muscle proportion for the animals of the other groups were also similar. The percentages of kidney and pelvic fat, related to EBW, were also similar among genetic groups but the carcass and EBW fat percentage were lower for Gi and Ca. The body composition estimates for the two kinds of equations were in agreement except for bone percentages in the Ca group.
\end{abstract}

Key Words: selection, body composition, Nellore, Gir, Guzerá, Caracu

\footnotetext{
1 Parte do projeto IZ-14-011/80

2 Estação Experimental de Zootecnia de Sertãozinho (SP). E.mail: razook@izsp.br

3 Instituto de Zootecnia - Nova Odessa (SP). E.mail: nardon@izsp.br

${ }^{4}$ Estudante de PhD - Cornell University - Ithaca - NY. E.mail: lot1@cornell.edu

${ }^{5}$ Bolsista CNPq.
} 


\section{Introdução}

O critério de seleção aplicado nos rebanhos da Estação Experimental de Zootecnia de Sertãozinho é o peso padronizado aos 378 dias (P378), em machos, obtido ao final de Provas de Ganho de Peso (PGP), e o peso padronizado aos 550 dias (P550), em fêmeas em regime de pasto. Vários estudos destacaram os ganhos genéticos alcançados nas características de crescimento de progênies Nelore e Guzerá (RAZOOK et al., 1993; RAZOOK et al., 1998) e também de Caracu, inserido na mesma metodologia de seleção (MERCADANTE et al. 1998). Dentro dessa linha de pesquisa e considerando-se que a progênie utilizada neste estudo já representa três gerações de seleção, tornou-se fundamental o acompanhamento das mudanças eventualmente ocorridas nas características quantitativas e qualitativas de carcaça. Alguns estudos já publicados mostram análises de características de carcaça envolvendo amostras populacionais nascidas em anos anteriores desses rebanhos (NARDON et al., 1998a,b; NARDON et al., 1999). Um dos efeitos indiretos mais importantes da seleção para peso pode ser a alteração na composição corporal dos bovinos e, nesse enfoque, NARDON (1998) avaliou os componentes físicos da carcaça por meio da dissecação completa da mesma, não tendo verificado alterações significativas nas porcentagens de músculo, gordura e osso entre animais dos rebanhos Nelore Seleção (altos diferenciais de seleção) e Controle (diferenciais nulos), embora tivesse havido entre outros grupos raciais (maior porcentagem de músculo e menor gordura no Caracu, por exemplo). Segundo NARDON et al. (1999), vários estudos têm demonstrado a possibilidade de estimação da composição física corporal ou da carcaça por meio da composição do corte entre a 9 $\underline{a}$ e $11^{\underline{a}}$ costelas, conforme procedimento de HANKINS e HOWE (1946).

Utilizando informações do corte entre a 9aa e $11^{\underline{a}}$ costelas e outras características de carcaça, NARDON (1998) desenvolveu equações estimadoras da composição física da carcaça de bovinos dos rebanhos Nelore, Guzerá e Caracu de Sertãozinho. Este estudo teve como objetivo fazer uso dessas equações para estimar a composição física da carcaça e do peso vazio de animais provenientes dos rebanhos selecionados para peso e pertencentes à $15^{\mathrm{a}}$ progênie resultante do projeto de melhoramento genético das raças Zebu e Caracu de Sertãozinho.

\section{Material e Métodos}

Os animais deste experimento foram amostras dos rebanhos Gir (Gi), Guzerá Seleção e Tradicional (GuS), Nelore Seleção e Tradicional (NeS) e Caracu $(\mathrm{Ca})$, todos selecionados para peso pósdesmame, e Nelore Controle $(\mathrm{NeC})$, não selecionado (diferenciais de seleção em torno de zero). Todos os animais dos cinco grupos foram nascidos em 1995 e participaram das Provas de Ganho de Peso (PGP) de 1996, de acordo com as normas descritas em RAZOOK et al. (1997). Ao final da referida prova, amostras constituídas de nove animais inteiros de cada rebanho (8 para GuS) foram separadas de modo a representar a média do peso padronizado aos 378 dias (P378) dos rebanhos. Os animais foram terminados em confinamento, sendo que o abate ocorreu a uma idade média de 580 dias e condição corporal 8 em uma escala de 1 a 9. Os detalhes do período de confinamento e do abate foram descritos por RAZOOK et al. (2001). Foram obtidos os pesos ao abate da carcaça e do fígado e, após o resfriamento das carcaças por 48 horas, retirou-se da $1 / 2$ carcaça esquerda uma seção transversal compreendendo as 9 $\stackrel{\mathrm{a}}{ }, 10^{\mathrm{a}}$ e $11^{\mathrm{a}}$ costelas, conforme procedimento de HANKINS e HOWE (1946). Esses cortes foram transportados ao laboratório de carnes do Instituto de Zootecnia, em Nova Odessa (SP), onde mediu-se a área do olho de lombo e a espessura de gordura na posição $11^{a}$ - 12 $\underline{\text { a }}$ costelas, além de separação e pesagem em seus componentes físicos: músculo, gordura e ossos.

Para a obtenção das estimativas de composição corporal, expressas em porcentagens de músculo, gordura e osso em relação ao peso vazio $(\mathrm{PVz})$ e ao peso da carcaça, foi estimado primeiramente o PVz pelas equações apresentadas por NARDON et al. (1998b), conforme Tabela 1. NARDON (1998) esclarece que o peso vazio é obtido na prática somando-se o peso da carcaça quente aos componentes extra-carcaça (sangue, couro, cabeça, patas, fígado, rins, outros órgãos, tecidos gastrintestinais livres de conteúdo, gordura renal e pélvica e outras gorduras cavitárias).

Para os cálculos de estimativas da quantidade $(\mathrm{kg})$ de músculo, gordura e osso da meia carcaça esquerda, foram utilizadas as equações de NARDON (1998), apresentadas na Tabela 2. Uma vez obtidas as quantidades em quilogramas 
Rev. bras. zootec.

Tabela 1 - Equações de regressão linear e coeficientes de determinação $\left(R^{2}\right)$ para estimativa do peso vazio $(\mathrm{PVz})$ por meio do peso vivo de abate (PAB) - geral e por rebanho

Table 1 - Empty body weight (EBW) predicting linear regression equations and determination coefficients based on slaughter weight (SW) - overall and by herd

\begin{tabular}{lccc}
\hline $\begin{array}{l}\text { Equação } \\
\text { Equation }\end{array}$ & $\begin{array}{c}\text { Intercepto } \\
\text { Intercept }\end{array}$ & $\begin{array}{c}\text { Coeficiente } \\
\text { PAB }(\mathrm{kg}) \\
\text { Coefficient } \\
\text { SW }(\mathrm{kg})\end{array}$ & $\mathrm{R}^{2}$ \\
\hline $\mathrm{PVz}_{\mathrm{Geral}}\left(E B W_{\text {overall }}\right)$ & $-15,320$ & 0,942 & 0,992 \\
$\mathrm{PVz}_{\mathrm{NeS}}\left(E B W_{\text {NeS }}\right)$ & $-21,697$ & 0,954 & 0,996 \\
$\mathrm{PVz}_{\mathrm{NeC}}\left(E B W_{N e C l}\right)$ & $-13,984$ & 0,945 & 0,992 \\
$\mathrm{PVz}_{\mathrm{GuS}}\left(E B W_{\mathrm{GuS}}\right)$ & $-17,962$ & 0,945 & 0,992 \\
$\mathrm{PVz}_{\mathrm{CaS}}\left(E B W_{\mathrm{CaS}}\right)$ & $-28,678$ & 0,962 & 0,992 \\
\hline
\end{tabular}

${ }_{1}^{1} \mathrm{NeS}$ : Nelore seleção; NeC: Nelore controle; GuS: Guzera Seleção e Ca: Caracu (NeS: Nellore selection; NeC: Nellore control; GuS: Guzera Selection and Ca:Caracu).

FONTE: NARDON et al. (1998b).

dos componentes, as mesmas foram transformadas em valores percentuais (estimativas 1) em relação à carcaça. Já para o cálculo das porcentagens de músculo, gordura e osso diretamente (estimativas 2) foram utilizadas as equações da Tabela 3. Essas estimativas da composição corporal foram também calculadas em relação ao peso vazio além das quantidades de gordura renal e o fígado. As estimativas da composição corporal dos animais do rebanho Gir foram feitas com base nas equações para o rebanho Nelore Controle, de peso semelhante. Esse procedimento foi adotado pela inexistência de equações para esse grupo genético.

As análises de variância, que incluíram basicamente o efeito fixo de rebanho, foram efetuadas pelo programa LSMLMW de HARVEY (1990) e os testes de significância das diferenças entre médias (Tukey), pelo SAS, Statistical Analysis System (1985).

\section{Resultados e Discussão}

Na Tabela 4 são apresentadas as médias observadas, bem como as suas comparações pelo teste de Tukey, para as estimativas de peso vazio (PVz) e de composição corporal, em termos de porcentagens de músculo (MCA), gordura (GCA) e osso (OCA) em relação à meia carcaça esquerda e também ao $\mathrm{PVz}$ (MPVz, GPVz e OPVz, respectivamente), para cada rebanho. São fornecidas as duas estimativas pelas equações encontradas em NARDON (1998) e NARDON et al. (1999). Nas estimativas identificadas por 1 , foram utilizadas as equações estimadoras da quantidade de músculo, gordura e osso da carcaça (Tabela 2) e, nas identificadas por 2, utilizaram-se as equações estimadoras de valores percentuais (Tabela 3). Constam da Tabela 4 os valores de pesos de abate (PAB), carcaça (PCAR), bem como os percentuais de músculo (MUSC), gordura (GORD) e osso (OSSO) encontrados no corte entre as $9^{\mathrm{a}}-11^{\mathrm{a}}$ costelas, apresentados por RAZOOK et al. (2001), os quais mantêm uma relação com a composição da carcaça e do peso vazio.

O PVz, em média, sofre redução de $9 \%$ em relação ao peso de abate (PAB). Isso está de acordo com o ocorrido no estudo de NARDON (1998), no qual o peso vazio foi obtido diretamente, e mostra que as equações estimaram com precisão essa variável, o que pode ser explicado pelos altos coeficientes de determinação. A exemplo do que ocorreu com PAB e PCAR, os animais dos rebanhos $\mathrm{CaS}, \mathrm{NeS}$ e $\mathrm{GuS}$ apresentaram $\mathrm{PVz}$ semelhantes e foram diferentes $(\mathrm{P}<0,01)$ dos animais do rebanho $\mathrm{NeC}$ que, por sua vez, não diferiram dos Gir.

Com relação à composição corporal, verifica-se que as estimativas 1 e 2 foram razoavelmente concordantes, embora para alguns rebanhos, como $\mathrm{Gi}$ e $\mathrm{Ca}$, as estimativas foram bem diferentes para porcentagem de músculo (MCA) e, principalmente, de osso na carcaça (OCA). Conseqüentemente, as estimativas em relação ao $\mathrm{PVz}$ também tiveram a mesma tendência (MPVz e OPVz). Aparentemente, as estimativas obtidas pela equação 2 foram as mais coerentes com as tendências da composição do corte das costelas, em termos de diferenças entre os grupos genéticos e também em relação ao estudo de NARDON (1998). Esse autor, no entanto, baseando-se nos maiores coeficientes de determinação, considerou as equações da Tabela 2, ou seja, as que estimam quantidades de músculo, gordura e osso, mais precisas.

De maneira geral, o efeito de rebanho foi significativo $(\mathrm{P}<0,01)$ para a maioria das variáveis estimadas, exceção feita à MPVz2 e porcentagem de gordura renal em relação a $\mathrm{PVz}$ (GRENPVz). No caso de MPVz2, os resultados estão de acordo com o encontrado por NARDON (1998), que não observou diferenças significativas, entre os grupos genéticos, na porcentagem de músculo separável da carcaça obtida direta- 
Tabela 2 - Equações estimadoras da quantidade $(\mathrm{kg})$ de músculo, gordura e osso da carcaça por rebanho Table 2 - Predicting equations of the amounts $(\mathrm{kg})$ of carcass muscle, bone and fat by herd

\begin{tabular}{|c|c|c|c|c|}
\hline \multirow[t]{2}{*}{$\mathrm{Y}^{(1)}$} & \multirow{2}{*}{$\begin{array}{l}\text { Intercepto } \\
\text { Intercept }\end{array}$} & \multicolumn{2}{|c|}{$\mathrm{X}^{(2)}$} & \multirow[t]{2}{*}{$\mathrm{R}^{2}$} \\
\hline & & $\begin{array}{l}\text { Variável } \\
\text { Variable }\end{array}$ & $\begin{array}{c}\text { Coeficiente } \\
\text { Coefficient }\end{array}$ & \\
\hline $\begin{array}{l}\operatorname{kgM}_{\mathrm{Ca}} \\
\operatorname{kgM}_{\mathrm{Ca}}\end{array}$ & 1,9050 & $\begin{array}{l}\operatorname{PCAR}(C W) \\
\operatorname{ESPGOR}(F T) \\
\operatorname{kgMUSC}(k g M U S C)\end{array}$ & $\begin{array}{r}0,28827 \\
-1,22381 \\
3,99524\end{array}$ & 0,9877 \\
\hline $\begin{array}{l}\mathrm{kgG}_{\mathrm{Ca}} \\
\mathrm{kg} G_{\mathrm{Ca}}\end{array}$ & $-4,1773$ & $\begin{array}{l}\text { ESPGOR }(F T) \\
\operatorname{kgMUSC}(k g M U S C) \\
\operatorname{kgGORD}(k g F A T)\end{array}$ & $\begin{array}{r}0,52021 \\
4,38567 \\
12,36473 \\
\end{array}$ & 0,9426 \\
\hline $\begin{array}{l}\mathrm{kgM}_{\mathrm{GuS}} \\
\operatorname{kgM}_{G u S}\end{array}$ & $-13,9157$ & $\begin{array}{l}\operatorname{PAB}(S W) \\
\operatorname{PVz}(E B W) \\
\operatorname{AOL}(L E A) \\
\operatorname{kgMUSC}(k g M U S C) \\
\operatorname{kgGORD}(k g F A T)\end{array}$ & $\begin{array}{r}0,04052 \\
0,12390 \\
0,23488 \\
7,59941 \\
-8,46045 \\
\end{array}$ & 0,9713 \\
\hline $\begin{array}{l}\mathrm{kgG}_{\mathrm{GuS}} \\
k g G_{G u S}\end{array}$ & $-11,6487$ & $\begin{array}{l}\operatorname{PAB}(S W) \\
\operatorname{PVz}(E B W) \\
\operatorname{kgMUSC}(k g M U S C) \\
\operatorname{kgGORD}(k g F A T) \\
\operatorname{kgOSSO}(\operatorname{kgBON})\end{array}$ & $\begin{array}{r}-0,00912 \\
0,11225 \\
-5,10976 \\
7,70354 \\
2,58464 \\
\end{array}$ & 0,9440 \\
\hline $\begin{array}{l}\operatorname{kgM}_{\mathrm{NeC}} \\
\operatorname{kgM}_{N e C}\end{array}$ & $-18,5840$ & $\begin{array}{l}\operatorname{PAB}(S W) \\
\operatorname{AOL}(L E A) \\
\operatorname{kgMUSC}(k g M U S C) \\
\operatorname{kgGORD}(k g F A T)\end{array}$ & $\begin{array}{r}0,17713 \\
0,26992 \\
6,67580 \\
-10,48274\end{array}$ & 0,9606 \\
\hline $\begin{array}{l}\mathrm{kgG}_{\mathrm{NeC}} \\
k g G_{\mathrm{NeC}}\end{array}$ & 1,2099 & $\begin{array}{l}\operatorname{PVz}(E B W) \\
\operatorname{AOL}(L E A) \\
\operatorname{kgGORD}(k g F A T)\end{array}$ & $\begin{array}{r}0,03865 \\
-0,07498 \\
14,49466 \\
\end{array}$ & 0,9818 \\
\hline $\begin{array}{l}\mathrm{kgM}_{\mathrm{NeS}} \\
\operatorname{kgM}_{N e S}\end{array}$ & $-8,8989$ & $\begin{array}{l}\operatorname{PCAR}(C W) \\
\operatorname{AOL}(L E A) \\
\operatorname{kgGORD}(k g F A T) \\
\operatorname{kgOSSO}(k g B O N)\end{array}$ & $\begin{array}{r}0,31130 \\
0,17335 \\
-9,82474 \\
10,42387 \\
\end{array}$ & 0,9788 \\
\hline $\begin{array}{l}\mathrm{kgG}_{\mathrm{NeS}} \\
k g G_{N e S}\end{array}$ & $-6,7250$ & $\begin{array}{l}\operatorname{PVz}(E B W) \\
\operatorname{AOL}(L E A) \\
\operatorname{kgGORD}(k g F A T)\end{array}$ & $\begin{array}{r}0,07424 \\
-0,09725 \\
9,63036 \\
\end{array}$ & 0,9621 \\
\hline \multicolumn{5}{|c|}{ 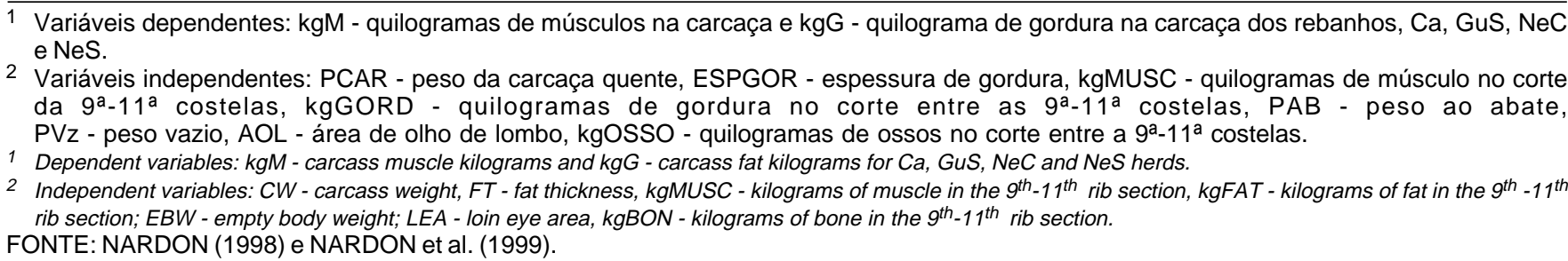 } \\
\hline
\end{tabular}

mente. Para músculo, os valores em relação ao $\mathrm{PVz}$ apresentados por esse autor oscilaram entre 39,1 $(\mathrm{GuS})$ e 40,0\% (Ca), portanto inferiores aos encontrados nesta pesquisa. Na comparação entre os animais Nelore Seleção e Controle, verifica-se que não houve efeitos indiretos da seleção para peso nas proporções de músculo, tanto na carcaça como no peso vazio estimado confirmando o encontrado no corte da costela. Nos outros grupos genéticos, Ca e Gi apresentaram maiores proporções de músculo pela estimativa 2 , tanto em relação à carcaça como ao $\mathrm{PVz}$. Com relação às porcentagens de gordura (GCA e
GPVz), os menores valores foram para os animais dos rebanhos Gir e Ca. No caso de GPVz1, o menor valor encontrado para os animais Ca diferiu significativamente $(\mathrm{P}<0,01)$ dos demais grupos genéticos. Esse resultado confirma o encontrado por NARDON (1998), embora esse autor tenha relatado porcentagens de gordura separável em relação ao $\mathrm{PVz}$ variando de 12,8 (Ca) a 16,0\% (NeC). Esses maiores valores de porcentagens de gordura podem ser atribuídos ao fato de que parte dos animais daquele estudo foi abatida em nível de terminação acima do ideal. Na comparação entre os animais $\mathrm{NeS}$ e $\mathrm{NeC}$, 
Rev. bras. zootec.

Tabela 3 - Equações estimadoras da porcentagem de músculo, gordura e osso da carcaça por rebanho Table 3 - Predicting equations of muscle, fat and bone percentages of carcass

\begin{tabular}{|c|c|c|c|c|}
\hline \multirow[t]{2}{*}{$\mathrm{Y}^{(1)}$} & \multirow{2}{*}{$\begin{array}{l}\text { Intercepto } \\
\text { Intercept }\end{array}$} & \multicolumn{2}{|c|}{$X^{(2)}$} & \multirow[t]{2}{*}{$\mathrm{R}^{2}$} \\
\hline & & $\begin{array}{l}\text { Variável } \\
\text { Variable }\end{array}$ & $\begin{array}{c}\text { Coeficiente } \\
\text { Coefficient }\end{array}$ & \\
\hline $\begin{array}{l}\mathrm{PM}_{\mathrm{Ca}} \\
M P_{C a}\end{array}$ & 70,4076 & $\begin{array}{l}\operatorname{AOL}(L E A) \\
\operatorname{ESPGOR}(F T) \\
\operatorname{GORD}(F A T)\end{array}$ & $\begin{array}{r}0,06101 \\
-0,48876 \\
-0,31122 \\
\end{array}$ & 0,9131 \\
\hline $\begin{array}{l}\mathrm{PG}_{\mathrm{Ca}} \\
P F A T_{C a}\end{array}$ & 48,0862 & $\begin{array}{l}\operatorname{PVz}(E B W) \\
\operatorname{PCAR}(C W) \\
\operatorname{AOL}(L E A) \\
\text { ESPGOR }(F T) \\
\operatorname{MUSC}(M U S) \\
\text { OSSO }(B O N)\end{array}$ & $\begin{array}{r}0,00766 \\
-0,00014 \\
-0,02168 \\
0,38027 \\
-0,37556 \\
-0,61079 \\
\end{array}$ & 0,9145 \\
\hline $\begin{array}{l}\mathrm{PG}_{\mathrm{GuS}} \\
P F A T_{G u S}\end{array}$ & 30,7245 & $\begin{array}{l}\operatorname{PAB}(S W) \\
\operatorname{PCAR}(C W) \\
\operatorname{MUSC}(M U S) \\
\operatorname{GORD}(F A T)\end{array}$ & $\begin{array}{r}0,00275 \\
0,01267 \\
-0,34528 \\
0,24288 \\
\end{array}$ & 0,8180 \\
\hline $\begin{array}{l}\mathrm{PO}_{\mathrm{GuS}} \\
P B O N{ }_{G u S}\end{array}$ & 43,6041 & $\begin{array}{l}\operatorname{PCAR}(C W) \\
\operatorname{AOL}(L E A) \\
\operatorname{MUSC}(M U S) \\
\operatorname{GORD}(F A T) \\
\end{array}$ & $\begin{array}{l}-0,01492 \\
-0,04189 \\
-0,26520 \\
-0,24882 \\
\end{array}$ & 0,8177 \\
\hline $\begin{array}{l}\mathrm{PG}_{\mathrm{NeC}} \\
P F A T_{N e C}\end{array}$ & 7,1502 & $\begin{array}{l}\operatorname{PCAR}(C W) \\
\operatorname{AOL}(L E A) \\
\operatorname{GORD}(F A T)\end{array}$ & $\begin{array}{r}0,00448 \\
-0,04043 \\
0,61622 \\
\end{array}$ & 0,8647 \\
\hline $\begin{array}{l}\mathrm{PO}_{\mathrm{NeC}} \\
P B O N N_{N e C} \\
\end{array}$ & 9,2681 & $\begin{array}{l}\operatorname{PVz}(E B W) \\
\operatorname{OSSO}(B O N)\end{array}$ & $\begin{array}{r}-0,00568 \\
0,49277 \\
\end{array}$ & 0,7851 \\
\hline $\begin{array}{l}\mathrm{PM}_{\mathrm{NeS}} \\
P M_{N e S} \\
\end{array}$ & 40,3598 & $\begin{array}{l}\operatorname{ESPGOR}(F T) \\
\operatorname{MUSC}(M U S)\end{array}$ & $\begin{array}{r}-0,15320 \\
0,41143 \\
\end{array}$ & 0,8517 \\
\hline $\begin{array}{l}\mathrm{PG}_{\mathrm{NeS}} \\
P F A T_{N e S}\end{array}$ & 8,3889 & $\begin{array}{l}\text { ESPGOR }(F T) \\
\text { MUSC }(M U S) \\
\operatorname{GORD}(F A T)\end{array}$ & $\begin{array}{r}0,14317 \\
-0,00711 \\
0,48126\end{array}$ & 0,9125 \\
\hline
\end{tabular}

1 Variáveis dependentes: PM - porcentagem de músculos, PG - porcentagem de gordura, PO - porcentagem de osso na carcaça de animais dos rebanhos $\mathrm{Ca}$, GuS, NeC e NeS

2 Variáveis independentes: AOL - área de olho de lombo, ESPGOR - espessura de gordura, GORD - \%gordura no corte da $9^{\mathrm{a}}-11^{\mathrm{a}}$ costelas, PVz - peso vazio, PCAR - peso da carcaça quente, MUSC - \%músculos no corte da 9ำ $11^{\text {a }}$ costelas, OSSO - \%ossos no corte da $9^{\mathrm{a}}-11^{\mathrm{a}}$ costelas, PAB - peso de abate.

${ }^{1}$ Dependent variables: MP - muscle percentage, PFAT - fat\%, PBON - bone \% of carcass of Ca , GuS, NeC and NeS herds.

2 Independent variables: LEA - loin eye area, FT - fat thickness, FAT - fat \% in the $9^{\text {th }}$ - $11^{\text {th }}$ rib section, EBW - empty body weight, CW - carcass weight MUS - muscle \% in the $9^{\text {th }}-11^{\text {th }}$ rib section, BON - bone \% in the $9^{\text {th }}-11^{\text {th }}$ rib section, SW - slaughter weight.

FONTE:NARDON (1998).

verifica-se também que não houve alterações significativas nas proporções de gordura na carcaça e no peso vazio estimado dos animais. As estimativas de porcentagens de osso (OCA e OPVz) são as que apresentaram maiores discrepâncias comparando-se os valores das duas equações, sendo que isso ocorreu principalmente com animais $\mathrm{Ca}$. Os valores para porcentagem de osso separável da carcaça no peso vazio, encontrados por NARDON (1998), oscilaram entre 9,5 (NeC) e 9,9\% (Ca), sendo que esses valores diferiram entre si significativamente. As estimativas de OPVz2 enquadram-se mais nesses limites, embora a tendência dos animais deste estudo é ter maior porcentagem de osso em relação àquele estudo de
NARDON (1998). Da mesma forma como ocorreu para músculo e gordura, as porcentagens de osso na carcaça e no PVz não sofreram modificações significativas decorrentes da seleção para peso, observadas na comparação entre $\mathrm{NeS}$ e $\mathrm{NeC}$. Com relação aos outros grupos genéticos, a comparação ficou prejudicada pelos resultados discrepantes das duas estimativas. Na Tabela 4 também são apresentadas as médias das porcentagens de gordura renal e pélvica, bem como do fígado em relação ao PVz. Contrariamente às quantidades de gordura renal, que diferiam significativamente entre os grupos genéticos, conforme relatado por RAZOOK et al. (2001), os valores percentuais não diferiram significativamente. Por outro lado, o fígado 
1042

RAZOOK et al.

Tabela 4 - Médias observadas de estimativas do peso vazio (PVz) e da composição corporal em relação ao PVz e carcaça, por rebanho

Table 4 - Observed means for estimated empty body weight (EBW) and body composition related to EBW and carcass weight

\begin{tabular}{|c|c|c|c|c|c|c|}
\hline \multirow[b]{2}{*}{$\begin{array}{l}\text { Variáveis } \\
\text { Variables } 1\end{array}$} & \multicolumn{6}{|c|}{$\begin{array}{c}\text { Rebanho }^{2} \\
\text { Herd }^{2}\end{array}$} \\
\hline & Gi & GuS & $\mathrm{NeC}$ & $\mathrm{NeS}$ & $\mathrm{Ca}$ & $\mathrm{NS}^{3}(L S)^{3}$ \\
\hline $\mathrm{PAB}(\mathrm{kg})(S W)$ & $407,7^{\mathrm{b}}$ & $493,7^{\mathrm{a}}$ & $425,9^{b}$ & $509,2^{\mathrm{a}}$ & $519,7^{\mathrm{a}}$ & 0,00 \\
\hline $\operatorname{PVz}(\mathrm{kg})(E B W)$ & $368,7^{\mathrm{b}}$ & $448,6^{\mathrm{a}}$ & $388,5^{\mathrm{b}}$ & $464,1^{\mathrm{a}}$ & $471,3^{\mathrm{a}}$ & 0,00 \\
\hline $\operatorname{PCAR}(\mathrm{kg})(\mathrm{CW})$ & $230,2^{b}$ & $274,5^{\mathrm{a}}$ & $247,6^{\mathrm{b}}$ & $292,1^{\mathrm{a}}$ & $289,7^{\mathrm{a}}$ & 0,00 \\
\hline$\overline{M U S C}(\%)(M U S)$ & $60,1^{b}$ & $59,8^{\mathrm{bc}}$ & $55,7^{d}$ & $56,4^{\mathrm{cd}}$ & $64,7^{\mathrm{a}}$ & 0,00 \\
\hline MCA1 (\%) (MCA1) & $66,8^{\mathrm{a}}$ & $63,8^{\mathrm{b}}$ & $63,7^{b}$ & $62,8^{\mathrm{b}}$ & $62,3^{\mathrm{b}}$ & 0,00 \\
\hline MCA2 (\%) (MCA2) & $65,0^{\mathrm{a}}$ & $64,5^{\mathrm{ab}}$ & $62,3^{b}$ & $62,5^{\mathrm{b}}$ & $66,2^{\mathrm{a}}$ & 0,00 \\
\hline$\overline{\operatorname{GORD}(\%)(F A T)}$ & $20,2^{\mathrm{c}}$ & $22,2^{b c}$ & $26,6^{\mathrm{a}}$ & $24,7^{\mathrm{ab}}$ & $18,7^{\mathrm{c}}$ & 0,00 \\
\hline $\operatorname{GCA} 1(\%)(F C A 1)$ & $18,4^{\mathrm{bc}}$ & $22,3^{\mathrm{a}}$ & $20,6^{\mathrm{ab}}$ & $21,5^{\mathrm{a}}$ & $16,3^{\mathrm{c}}$ & 0,00 \\
\hline GCA2 $(\%)(F C A 2)$ & $18,1^{\mathrm{b}}$ & $20,3^{\mathrm{ab}}$ & $21,9^{\mathrm{a}}$ & $21,1^{\mathrm{a}}$ & $17,9^{\mathrm{b}}$ & 0,00 \\
\hline $\operatorname{OSSO}(\%)(B O N)$ & $19,6^{\mathrm{a}}$ & $17,9^{\mathrm{ab}}$ & $17,7^{\mathrm{ab}}$ & $18,9^{\mathrm{a}}$ & $16,7^{b}$ & 0,00 \\
\hline OCA1 $(\%)(B C A 1)$ & $14,8^{\mathrm{b}}$ & $14,0^{\mathrm{b}}$ & $15,7^{\mathrm{b}}$ & $15,7^{\mathrm{b}}$ & $21,4^{\mathrm{a}}$ & 0,00 \\
\hline OCA2 $(\%)(B C A 2)$ & $16,9^{\mathrm{a}}$ & $15,2^{\mathrm{b}}$ & $15,8^{\mathrm{ab}}$ & $16,4^{\mathrm{a}}$ & $15,9^{\mathrm{ab}}$ & 0,00 \\
\hline$\overline{M P V z 1(\%)(M E B W 1)}$ & $42,1^{\mathrm{a}}$ & $39,0^{\mathrm{bc}}$ & $40,6^{\mathrm{ab}}$ & $39,5^{\mathrm{bc}}$ & $38,3^{\mathrm{c}}$ & 0,00 \\
\hline $\operatorname{MPVz} 2(\%)(M E B W 2)$ & $40,9^{\mathrm{a}}$ & $39,3^{\mathrm{a}}$ & $39,4^{\mathrm{a}}$ & $39,3^{\mathrm{a}}$ & $40,6^{\mathrm{a}}$ & 0,10 \\
\hline GPVz1 $(\%)(F E B W 1)$ & $11,6^{\mathrm{b}}$ & $13,6^{\mathrm{a}}$ & $13,1^{\mathrm{ab}}$ & $13,5^{\mathrm{a}}$ & $10,0^{\mathrm{c}}$ & 0,00 \\
\hline $\operatorname{GPVz} 2(\%)(F E B W 2)$ & $11,4^{\mathrm{b}}$ & $12,4^{\mathrm{ab}}$ & $13,9^{\mathrm{a}}$ & $13,3^{\mathrm{a}}$ & $11,0^{\mathrm{b}}$ & 0,00 \\
\hline OPVz1 (\%)(BEBW1) & $9,4^{\mathrm{b}}$ & $8,6^{\mathrm{b}}$ & $10,0^{\mathrm{b}}$ & $9,9^{\mathrm{b}}$ & $13,1^{\mathrm{a}}$ & 0,00 \\
\hline OPVz2 $(\%)(B E B W 2)$ & $10,6^{\mathrm{a}}$ & $9,2^{\mathrm{c}}$ & $10,0^{\mathrm{ab}}$ & $10,3^{\mathrm{ab}}$ & $9,8^{\mathrm{bc}}$ & 0,00 \\
\hline$\overline{\operatorname{GRENPVz}(\%)(K I F E B W)}$ & $2,2^{\mathrm{a}}$ & $2,5^{\mathrm{a}}$ & $2,6^{\mathrm{a}}$ & $2,4^{\mathrm{a}}$ & $2,1^{\mathrm{a}}$ & 0,16 \\
\hline $\operatorname{FIGPVz}(\%)(L I V E B W)$ & $1,3^{\mathrm{ab}}$ & $1,3^{\mathrm{ab}}$ & $1,2^{b}$ & $1,3^{\mathrm{ab}}$ & $1,4^{\mathrm{a}}$ & 0,01 \\
\hline \multicolumn{7}{|c|}{$\begin{array}{l}1 \text { PAB: peso de abate; PVz: peso vazio estimado; PCAR: Peso de carcaça; MUSC: porcentagem de músculo no corte; MCA1 e MCA2: porcentagens } \\
\text { estimadas de músculo na carcaça (estimativas } 1 \text { e 2, respectivamente); GORD: porcentagem de gordura no corte; GCA1 e GCA2: porcentagens } \\
\text { estimadas } 1 \text { e } 2 \text { de gordura na carcaça respectivamente; OSSO: porcentagem de osso no corte; OCA1 e OCA2: porcentagens estimadas de } \\
\text { osso na carcaça; MPVz, GPVz e OPVZ: porcentagens de músculo, gordura e osso em relação ao peso vazio, conforme equações } 1 \text { e 2; GRENPVz } \\
\text { e FIGPVz: porcentagens de gordura renal e fígado em relação ao PVz. } \\
2 \text { Gi: Gir; GuS: Guzerá (seleção e tradicional); NeC: Nelore controle; NeS: Nelore (seleção e tradicional); Ca: Caracu. } \\
{ }^{3} \text { Nível de significância. }\end{array}$} \\
\hline \multicolumn{7}{|c|}{$\begin{array}{l}1 \text { SW:slaughter weight; EBW: estimated empty body weight; } C W \text { : carcass weight; MUS: muscle percentage in the rib section; MCA1 and MCA2: carcass muscle percentage } \\
\text { estimates (1 and } 2 \text { respectively); FAT: fat percentage in the rib section; FCA1 and FCA2: carcass fat percentages estimates } 1 \text { and 2; BON: bone percentage in the } \\
\text { rib section; BCA1 and BCA2: carcass bone percentages estimates; MEBW, FEBW and BEBW: muscle, fat and bone percentages related to EBW by estimates } 1 \text { and } 2 \text {; } \\
\text { KIFEBW and LIVEBW: percentages of kidney fat and liver related to EBW. }\end{array}$} \\
\hline $\begin{array}{l}2 \text { Gi: Gyr; GuS: Guzera (selection } \\
3 \text { Significance level. }\end{array}$ & ditional); $\mathrm{Ne}$ & Nellore: $\mathrm{Ne}$ & and tradition & & & \\
\hline
\end{tabular}

de animais Caracu foi maior que dos outros grupos genéticos em relação ao peso vazio.

Os valores das estimativas de composição corporal encontrados neste estudo devem ser considerados com restrições. Em primeiro lugar, foram oriundas de equações obtidas de animais abatidos em três pontos diferentes de terminação (antes, no ponto ideal e acima do grau de acabamento adequado). Em segundo, convém destacar que, para as estimativas do Gir, foram utilizadas as equações do Nelore Controle $(\mathrm{NeC})$, que, embora tenham sido estimadas para animais de tamanho e peso semelhantes, podem não necessariamente representar a composição corporal desse rebanho. Há necessidade, portanto, de novos estudos que avaliem a composição total da carcaça, e não somente do corte da costela desses grupos genéticos, e mesmo de outros que possibilitem consolidar os componentes dessas equações.

\section{Conclusões}

A seleção com base em pesos pós-desmame, visualizada na comparação entre animais $\mathrm{NeS}$ em relação ao $\mathrm{NeC}$, não promoveu alterações indiretas nas proporções de músculo, gordura e osso tanto na carcaça como no peso vazio dos animais.

As proporções de músculo nos outros grupos genéticos foram semelhantes às encontradas nos 
animais Nelore, porém animais Ca e Gi apresentaram menores proporções de gordura na carcaça e no peso vazio.

As quantidades de gordura renal e pélvica, transformadas em percentuais de peso vazio, também não foram diferentes entre os vários grupos genéticos.

As estimativas de composição corporal, resultantes dos dois tipos de equações foram razoavelmente concordantes, diferindo somente para porcentagem de ossos principalmente em animais Ca.

\section{Referências Bibliográficas}

HANKINS, O.G., HOWE, P.E. 1946. Estimation of the composition of beef carcass and cuts. United States Department of Agriculture. p.20 (Technical Bulletin ).

HARVEY, W.R. 1990. User's guide for LSMLMW (Mixed Model Least-Squares and Maximum Likelihood Computer Program). Wooster: Ohio State University. 91p.

MERCADANTE, M.E.Z., FIGUEIREDO, L.A. de, TROVO, J.B. de F. et al. Estimativas de parâmetros e mudança genética em características de crescimento do rebanho Caracu de Sertãozinho. In: REUNIÃO ANUAL DA SOCIEDADE BRASILEIRA DE ZOOTECNIA, 35, 1998. Botucatu. SP. Anais... Botucatu: SBZ, 1998. v.3, p.380-382.

NARDON, R.F. Seleção de bovinos para desempenho: composição corporal e características de carcaça. Jaboticabal, SP: FCAV, 1998. 107p. Tese (Doutorado em Zootecnia) - Universidade Estadual Paulista, 1998.

NARDON, R.F., TEDESCHI, L.O., BOIN, C. et al. Growth performance and carcass composition of bos indicus and adapted bos taurus selected based on weight gain after weaning and on adjusted weight at 378 days of age. In: WORLD CONGRESS ON GENETICS APPLIED TO LIVESTOCK PRODUCTION, 6., 1998, Armidale, Proceedings...Armidale, 1998a. p.137-140.

NARDON, R.F., RAZOOK, A.G., SAMPAIO, A.A.M. et al. Efeito da seleção para peso pós-desmama e de raças no rendimento em cortes da carcaça e na qualidade da carne de bovinos. In: REUNIÃO ANUAL DA SOCIEDADE BRASILEIRA DE ZOOTECNIA, 35., 1998, Botucatu. Anais... Botucatu: SBZ, 1998b, p.362-364.
NARDON, R.F., SAMPAIO, A.A.M., RAZOOK, A.G. et al. Equações de estimativa da quantidade de músculos e de gordura na carcaça de garrotes zebuínos e taurinos. In: REUNIÃO ANUAL DA SOCIEDADE BRASILEIRA DE ZOOTECNIA, 36., 1999, Porto Alegre. Anais... Porto Alegre: SBZ, 1999. p.333.

RAZOOK, A.G., FIGUEIREDO, L.A., TROVO, J.B. et al. 1993. Intensidades de seleção e respostas direta e correlacionadas em 10 anos de progênies de bovinos das raças Nelore e Guzerá selecionadas para peso pós-desmame. Bol. Ind. Anim., 50(2):147-163.

RAZOOK, A.G., FIGUEIREDO, L.A., CYRILLO, J.N.S.G. et al. 1997. Prova de ganho de peso. Normas adotadas pela Estação Experimental de Zootecnia de Sertãozinho. Instituto de Zootecnia. p.42 (Boletim Técnico no 40).

RAZOOK, A.G., FIGUEIREDO, L.A., BONILHA NETO, L.M. et al. 1998. Selection for yearling weight in Nelore and Guzerá zebu breeds: selection applied and response in 15 years of progeny. In. WORLD CONGRESS ON GENETICS APPLIED TO LIVESTOCK PRODUCTION, 6., 1998, Armidale. Proceedings...Armidale, 1998. p.133-136.

RAZOOK, A.G., FIGUEIREDO, L.A., NARDON, R.F. et al. 2001. Efeitos da raça e da seleção para peso pós-desmame sobre características de confinamento e de carcaça da $15^{\mathrm{a}}$ progênie dos rebanhos Zebu e Caracu de Sertãozinho (SP). Rev. bras. zootec., 30(1):115-124.

SAS Institute Inc. 1985. SAS Introductory Guide for Personal Computer. Version 6.ed, Cary, NC; SAS Institute. 111p.

Recebido em: 26/09/00

Aceito em: 29/01/01 